\title{
Usefulness of Measuring Airway Length with Cephalometry in Pediatric Subjects with Obstructive Sleep Apnea
}

\author{
Jae Hyung Hwang, $\mathrm{MD}^{1}$, Dong Sun Park, $\mathrm{MD}, \mathrm{PhD}^{2}$, In Hye Kim, $\mathrm{MD}^{1}$, \\ Hyesook Lee, $\mathrm{MD}^{1}$ and Chan-Soon Park, $\mathrm{MD}, \mathrm{PhD}^{1}$ \\ ${ }^{I}$ Department of Otolaryngology-Head and Neck Surgery, St. Vincent's Hospital, College of Medicine, The Catholic University of Korea, \\ Suwon; and ${ }^{2}$ Ds Sleep Center, Seoul, Korea
}

Background and Objectives: This study was designed to analyze the relationship between measurement of airway length with cephalometry and $\mathrm{AHI}$ in children with OSA and to assess the correlations between cephalometric parameters and salivary cortisol level.

Subjects and Method: Three parameters of the upper airway were measured by cephalometry: distance from the posterior nasal spine to the hyoid bone (PHy), distance from the mandibular plane to the hyoid bone (MP-Hy), and distance from the posterior nasal spine to the mandibular plane (P-MP). Ratios for each segment were also determined.

Results: Subjects with OSAS had longer P-Hy and MP-Hy than the control group. In particular, MP-Hy was significantly longer in the moderate-severe group than the other groups. In the OSAS group, there were significant correlations between MP-Hy, Ratio1, Ratio3 and m-Cor, s-Cor.

Conclusion: Length of upper airway and ratio parameters of cephalometry may be more useful indices in moderate-severe OSA than absolute distance parameters, and Ratio1 may be valuable for prediction of activating HPA axis.

KEY WORDS: Sleep apnea, obstructive $\cdot$ Cephalometry $\cdot$ Child $\cdot$ Airway resistance.

\section{INTRODUCTION}

Obstructive sleep apnea syndrome (OSAS) is characterized by repeated episodes of partial or complete cessation of airflow during sleep, and is a relatively common disorder with a prevalence of $1 \%$ to $6 \%$ in pediatric subjects. ${ }^{1-3)}$ Thus, the American Academy of Pediatrics recommends screening all snoring children for OSAS. ${ }^{1)}$ The gold standard for the diagnosis of OSAS remains overnight polysomnography (PSG), but it is not routinely performed for all suspicious pediatric subjects because of its expense, timeconsuming nature, and the high cure rate by adenotonsillectomy. To date, no standard screening protocol for OSAS has been developed in pediatrics. Although a few diagnostic methods such as questionnaires and physical examinations have been studied, their diagnostic accuracy has not been demonstrated or compared to a standard diagnostic method. ${ }^{4}$

The human larynx is thought to descend from its original position along with the development of phonation or speech in human evolution, resulting in elongation of the upper airway. This pharyngolaryngeal elongation may have increased the risk for obstructive sleep apnea (OSA), ${ }^{5)}$ because a pharyngolaryngeal segment without outer skeletal supports is known as the main cause of airway collapse in OSA. Assuming that the upper airway in humans is cylindrical in

Received: November 16, 2018 / Revised: February 27, 2019 / Accepted: April 11, 2019

Address for correspondence: Chan-Soon Park, MD, PhD, Department of Otorhinolaryngology-Head and Neck Surgery, St. Vincent's Hospital, College of Medicine, The Catholic University of Korea, 93 Jungbu-daero, Paldal-gu, Suwon 16247, Korea

Tel: +82-31-881-8968, Fax: +82-31-257-3752, E-mail: pcs0112@catholic.ac.kr 
shape, the length and radius of the pharyngeal segment could affect resistance to airflow, according to Poiseuille's law. In other words, an increase in the length of the pharyngeal segment would be expected to increase the possibility of the development of OSA. It is difficult to measure of radius of the human pharyngeal segment and its changes according to inspiration and expiration, but the length of the pharyngeal segment is relatively consistent. Cephalometry is an inexpensive and non-invasive technique that measures the pharyngolaryngeal length. Although cephalometric analysis has been used to evaluate the obstructive upper airway, there are many parameters to measure and no standard parameters to diagnosis OSA.

The objective of this study is to evaluate the relationship between cephalometric pharyngolaryngeal length and OSA, investigating which cephalometric parameters can be more reliable indices.

\section{SUBJECTS AND METHOD}

\section{Subjects}

This study subjects were 102 children, enrolled retrospectively, who underwent PSG for sleep evaluation at the Department of Otolaryngology and Head and Neck Surgery (St. Vincent's Hospital, Suwon, Republic of Korea) between January 2011 and December 2012. The study protocol was reviewed and approved by the Ethics Committee for Clinical Studies at St. Vincent's Hospital, The Catholic University of Korea. All subjects underwent thorough otorhinolaryngological examinations and full, attended overnight polysomnography. We excluded 52 subjects who did not undergo cephalometry, and 11 subjects who were obese (BMI $>25 \mathrm{~kg} / \mathrm{m}^{2}$ or BMI $>95$ th percentile). In total, 39 children with no craniofacial anomaly and no sleep disorder except OSA were included. Tonsil sizes were measured according to the Brodsky tonsil index, and adenoid sizes were measured as described by Fujioka et al. ${ }^{\text {) }}$

\section{Polysomnography}

All methods and equipment used in this study for PSG were used as described in our previous reports. ${ }^{788}$ Briefly, full, attended overnight PSG was performed in all participants using a computerized PSG system (Somnologica software and Embla S700/A10 hardware; Embla Systems Inc., Broomfield, CO, USA). Physiological signals were recorded with four EEG channels, two EOG channels, one ECG- lead, three EMGs (chin, anterior tibialis muscles, right and left), and one body position sensor. Respiratory signals were monitored with a nasal pressure transducer, an oral thermistor, a thoracic and abdominal respiratory effort sensor (Piezo type), a pulse oxymeter, and a neck vibration sensor for snoring. All measured parameters were interpreted manually by a certified technician and were reviewed by a doctor.

Apnea was defined as the absence or reduction of $>90 \%$ of oronasal airflow for $>90 \%$ of the entire event for a period lasting at least two regular breaths. Hypopnea was defined as a $50 \%$ or greater air flow reduction for $>90 \%$ of the entire event lasting for at least two regular breaths, with a desaturation of $3 \%$ or greater, awakening, or EEG arousal. The AHI was defined as the number of apnea and hypopnea episodes per hour of total sleep time. OSA in pediatric subjects was defined as AHI $>1$.

\section{Cephalometry}

Lateral cephalometry was obtained using standard techniques in an upright position during the end-expiratory phase. Bilateral ear rods were inserted into patient's external ear canal, and the patient's forehead was placed on a headrest to maintain the Frankfort plane parallel to the ground. All subjects were asked to keep their teeth and jaws in centric relation, and to close their lips and teeth naturally. Initially, we measured three distances, but because the absolute value of the length may not reflect OSAS because growth in children varies by individual, three parameter ratios from the three distance parameters were also calculated. The reference point and parameters used are shown in Fig. 1 and Table 1.

\section{Statistical analyses}

Statistical analyses were conducted using SPSS software (SPSS Inc., Chicago, IL, USA). After the Shapiro-Wilk test was performed, a parametric statistical analysis was performed for variables that followed a normal distribution, and non-parametric statistics were used for variables that did not follow a normal distribution. Quantitative variables in the OSAS (AHI $>1)$ and control (AHI $\leq 1)$ groups were compared using the Student's t-test, Wilcoxon rank sum test, and $\chi^{2}$ test. ANOVA and the Kruskal-Wallis test, followed by a post-hoc Dunn's test were performed to investigate the relationship between cephalometric parameters and OSAS severity among the control group and each OSAS 
subgroup. Receiver operating characteristic (ROC) curves were used to determine the sensitivity and specificity associated with a given cut-off of the cephalometric parameters

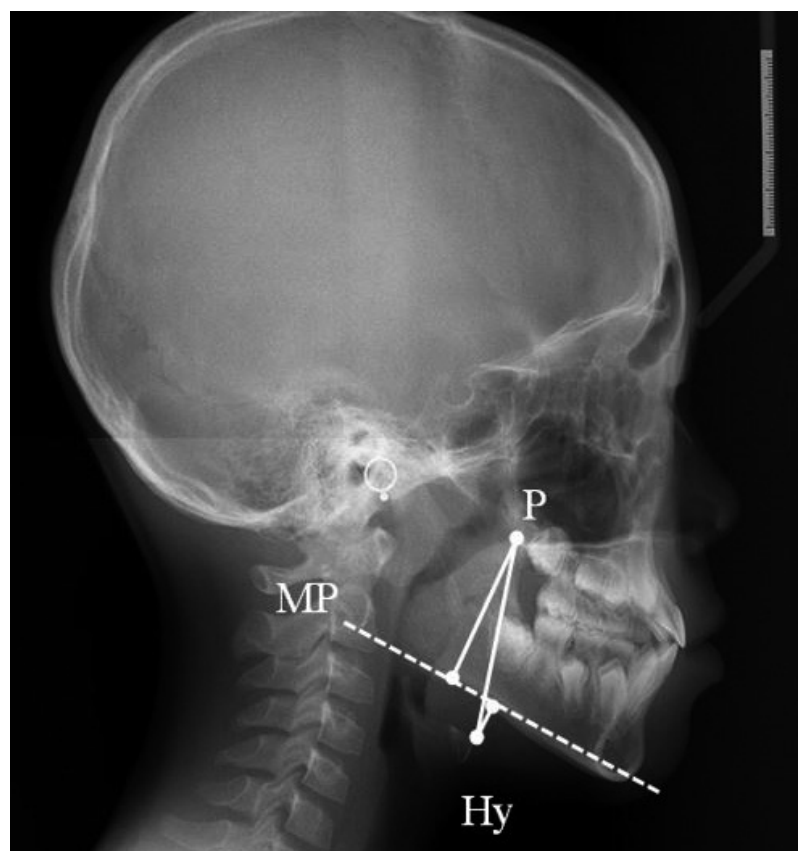

Fig. 1. Cephalometric landmarks and parameters. P: posterior nasal spine, MP: mandibular plane, $\mathrm{Hy}$ : the most anterior and superior point of hyoid bone.

Table 1. Definition of the cephalometric parameters

\begin{tabular}{ll} 
Parameters & \multicolumn{1}{c}{ Definition } \\
\hline P-Hy & $\begin{array}{l}\text { The distance from posterior nasal spine to hyoid } \\
\text { bone } \\
\text { The distance from mandibular plane to hyoid } \\
\text { bone }\end{array}$ \\
P-MP & $\begin{array}{l}\text { The distance from posterior nasal spine to } \\
\text { madibular plane }\end{array}$ \\
Ratio 1 & The ratio of $\mathrm{M}-\mathrm{H} / \mathrm{H}-\mathrm{P}$ \\
Ratio 2 & The ratio of $\mathrm{P}-\mathrm{M} / \mathrm{H}-\mathrm{P}$ \\
Ratio 3 & The ratio of $\mathrm{M}-\mathrm{H} / \mathrm{P}-\mathrm{M}$
\end{tabular}

and in terms of predicting a positive PSG. Univariable logistic regression analysis was used to calculate the odds ratio (OR).

\section{RESULTS}

The subjects $(n=39)$ were divided into control $(n=18$, AHI $\leq 1)$ and OSAS $(n=21$, AHI $>1)$ groups. The demographic data and PSG finding are presented in Table 2. The ratio of males:females in the OSAS group (17 males, 80.9\%; 4 females, 19.1\%) differed from that of the control group (9 males, $50 \%$; 9 females, 50\%). There were no significant differences in BMI between the groups. The mean age of the OSAS group $(5.9 \pm 2.2)$ was younger than the control group (7.2 \pm 1.9$)$, although not statistically significant $(\mathrm{p}=0.06)$. Thus, we adjusted the $\mathrm{p}$-values for age and gender.

As shown in Table 3, tonsil size and adenoid ratio did not differ between the groups. The PHy and MP-Hy in the OSAS group were significantly longer than in the control group. However, there were no significant differences in P-MP between groups. Ratio 2 was lower, and Ratios 1 and 3 were higher in the OSAS group after adjusting for age and gender.

Table 2. The demographic data and PSG finding of studied patients

\begin{tabular}{lccc} 
& $\begin{array}{c}\text { Control } \\
\text { AHI } \leq 1(n=18)\end{array}$ & $\begin{array}{c}\text { OSAS } \\
\text { AHI }>1(n=21)\end{array}$ & P value \\
\hline Gender & & & \\
$\quad$ Male & $9(50.0)$ & $17(80.9)$ & 0.04 \\
Female & $9(50.0)$ & $4(19.1)$ & \\
Age & $7.2 \pm 1.9$ & $5.9 \pm 2.2$ & 0.06 \\
BMI & $16.9 \pm 2.3$ & $16.6 \pm 2.3$ & 0.58 \\
AHI & $0.2 \pm 0.3$ & $11.1 \pm 9.8$ & $<0.0001$ \\
\hline
\end{tabular}

Data are presented as mean \pm SD; as $n(\%)$. $p$ value difference between control and OSAS by Chi-square test, Student $\dagger$ test and wilcoxon rank sum test

Table 3. Comparison of physical and cephalometric parameters in patients with OSAS and control group

\begin{tabular}{lcccc} 
& $\begin{array}{c}\text { Control } \\
\mathrm{AHI} \leq 1(\mathrm{n}=18)\end{array}$ & $\begin{array}{c}\text { OSAS } \\
\mathrm{AHI}>1(\mathrm{n}=21)\end{array}$ & P value* $^{*}$ & Adjusted OR $(95 \% \mathrm{Cl}) / \mathrm{P}$ value $^{\dagger}$ \\
\hline Tonsil & $2.8 \pm 0.9$ & $3.2 \pm 0.6$ & 0.0954 & $2.017(0.727-5.598) / 0.1780$ \\
AN-r & $0.6 \pm 0.1$ & $0.6 \pm 0.1$ & 0.0550 & $59.163(0.014-\mathrm{inf}) / 0.3363$ \\
P-Hy & $51.8 \pm 5.5$ & $56.1 \pm 5.5$ & 0.0217 & $1.334(1.075-1.655) / 0.0089$ \\
MP-Hy & $6.7 \pm 3.2$ & $10.4 \pm 5.5$ & 0.0358 & $1.271(1.021-1.581) / 0.0316$ \\
P-MP & $41.3 \pm 4.1$ & $40 \pm 3.4$ & 0.2827 & $0.976(0.782-1.219) / 0.8334$ \\
Ratio 1 & $0.1 \pm 0.1$ & $0.2 \pm 0.1$ & 0.0502 & $\operatorname{Inf}(1.440-\operatorname{Inf}) / 0.0437$ \\
Ratio 2 & $0.8 \pm 0.1$ & $0.7 \pm 0.1$ & 0.0004 & $\operatorname{Inf}(\operatorname{Inf}-0.009) / 0.0106$ \\
Ratio 3 & $0.2 \pm 0.1$ & $0.3 \pm 0.1$ & 0.0270 & $\operatorname{Inf}(2.172-\operatorname{Inf}) / 0.0334$ \\
\hline
\end{tabular}

*: p values of significant difference between Control and OSAS, by Student † test and Wilcoxon rank sum test, $\dagger: p$ values of difference between Control and OSAS by multivariable logistic regression (adjust: age and gender). Inf: infinity 
Ratio 1 positively correlated with AHI in the OSAS group $(\mathrm{r}=0.445, \mathrm{p}=0.0430$; Table 4). Although there was no statistically significant difference, MP-Hy and Ratio 3 showed a positively correlated trend $(\mathrm{r}=0.3987, \mathrm{p}=0.0734$ and $\mathrm{r}=$ $0.3896, \mathrm{p}=0.0808$, respectively).

After dividing the OSAS group into mild $(1<\mathrm{AHI} \leq 5)$ and moderate-severe (AHI $>5$ ) severity, P-Hy was significantly longer in the moderate-severe group than in the con-

Table 4. The relationship between AHI and physical and cephalometric parameters in OSAS group

\begin{tabular}{lcc} 
& \multicolumn{2}{c}{$\mathrm{AHI}$} \\
\cline { 2 - 3 } & $\mathrm{r}$ & $\mathrm{P}$ value \\
\hline Tonsil & 0.0788 & 0.7342 \\
AN-r & 0.3437 & 0.1272 \\
P-Hy & 0.0249 & 0.9147 \\
MP-Hy & 0.3987 & 0.0734 \\
P-MP & 0.0085 & 0.9709 \\
Ratio 1 & 0.4455 & 0.0430 \\
Ratio 2 & 0.0104 & 0.9642 \\
Ratio 3 & 0.3896 & 0.0808
\end{tabular}

Statistics were carried out using Pearson's and Spearman's correlation analysis trol group (Table 5). Additionally, MP-Hy was significantly longer in the moderate-severe group than in the control and mild groups. Ratios 1 and 3 were higher in the moderate-severe group than in the other two groups, and Ratio 2 was lower in the moderate-severe group than in the control group. There was no statistically significant difference between the control and mild groups for any parameter.

ROC curves were constructed; the area under the curves represented the discriminatory power of each parameter in predicting moderate-severe OSAS (Table 6). Almost all parameters showed statistical significance, except tonsil size and P-MP parameters. In particular, P-Hy, MP-Hy, and Ratios 1, 2, and 3 showed high areas under the curve compared to AN-r. Although the sensitivities of ratio parameters (Ratios 1, 2, and 3) were lower than those of absolute distance parameters (P-Hy, MP-Hy, and P-MP), the specificities of ratio parameters were more than $90 \%$.

Univariate logistic regression analysis showed that the ORs of ratio parameters were higher than those of absolute distance parameters (Table 7). The ratio parameters had higher ORs ( -3 fold) than the absolute parameters. This in-

Table 5. Comparison of the physical and cephalometric parameters according to the OSA severity

\begin{tabular}{|c|c|c|c|c|c|c|}
\hline & \multirow{2}{*}{$\begin{array}{l}\text { Control } \\
\text { AHI } \leq 1 \\
(n=18)\end{array}$} & \multirow{2}{*}{$\begin{array}{c}\text { Mild } \\
1<\text { AHI } \leq 5 \\
(n=7)\end{array}$} & \multirow{2}{*}{$\begin{array}{c}\text { Moderate-severe } \\
5<\mathrm{AHI} \\
(n=14)\end{array}$} & \multirow[b]{2}{*}{$p$ value* } & \multicolumn{2}{|c|}{ Event=moderate severe } \\
\hline & & & & & Control OR $(95 \% \mathrm{Cl}) / \mathrm{p}$ value $^{\dagger}$ & Mild OR $(95 \% \mathrm{Cl}) / \mathrm{p}$ value ${ }^{\dagger}$ \\
\hline Tonsil & $2.8 \pm 0.9$ & $3.1 \pm 0.7$ & $3.3 \pm 0.6$ & 0.2196 & $2.333(0.740-7.355) / 0.1481$ & $1.592(0.366-6.913) / 0.5350$ \\
\hline AN-r & $0.6 \pm 0.1$ & $0.6 \pm 0.1$ & $0.7 \pm 0.1$ & 0.1340 & $343.512(0.039-\operatorname{lnf}) / 0.2080$ & $295.429(0.005-\operatorname{lnf}) / 0.3077$ \\
\hline $\mathrm{P}-\mathrm{Hy}$ & $51.8 \pm 5.5$ & $53.6 \pm 3.8$ & $57.3 \pm 6$ & 0.0273 & $1.413(1.108-1.803) / 0.0054$ & $1.158(0.931-1.441) / 0.1883$ \\
\hline MP-Hy & $6.7 \pm 3.2$ & $6.9 \pm 3.5$ & $12.2 \pm 5.6$ & 0.0089 & $1.413(1.086-1.838) / 0.0101$ & $1.328(1.006-1.753) / 0.0453$ \\
\hline P-MP & $41.3 \pm 4.1$ & $40.6 \pm 1.8$ & $39.7 \pm 4$ & 0.4829 & $0.932(0.733-1.186) / 0.5691$ & $0.846(0.610-1.175) / 0.3185$ \\
\hline Ratio 1 & $0.1 \pm 0.1$ & $0.1 \pm 0.1$ & $0.2 \pm 0.1$ & 0.0126 & $\operatorname{Inf}(75.773-\operatorname{Inf}) / 0.0122$ & $\operatorname{lnf}(3.127-\operatorname{lnf}) / 0.0364$ \\
\hline Ratio 2 & $0.8 \pm 0.1$ & $0.8 \pm 0.1$ & $0.7 \pm 0.1$ & 0.0002 & $\operatorname{lnf} / 0.0046$ & $\operatorname{lnf}(\operatorname{Inf}-4.014) / 0.0719$ \\
\hline Ratio 3 & $0.2 \pm 0.1$ & $0.2 \pm 0.1$ & $0.3 \pm 0.1$ & 0.0074 & $\operatorname{Inf}(26.861-\operatorname{Inf}) / 0.0114$ & $\operatorname{lnf}(1.122-\operatorname{Inf}) / 0.0478$ \\
\hline
\end{tabular}

*: p value difference between Control, Mild and Moderate-Severe by ANOVA and Kruskal-Wallis test, followed by post hoc scheffe and Dunn's test, $\dagger: p$ values of difference between Control, Mild and Moderate-Severe by multivariable logistic regression (adjust: age and gender)

Table 6. The evaluation of the effective screening measurements to identify the moderate-severe OSAS group in all patients

\begin{tabular}{lccccc}
\hline & Cut-off value & Sen $(95 \% \mathrm{Cl})$ & Spe $(95 \% \mathrm{Cl})$ & AUC $(95 \% \mathrm{Cl})$ & $\mathrm{P} \mathrm{value}$ \\
\hline Tonsil & $>2$ & $92.86(66.1-99.8)$ & $24.00(9.4-45.1)$ & $0.630(0.461-0.779)$ & 0.1109 \\
AN-r & $>0.6$ & $78.57(49.2-95.3)$ & $68.00(46.5-85.1)$ & $0.683(0.514-0.822)$ & 0.0381 \\
P-Hy & $>52.69$ & $78.57(49.2-95.3)$ & $64.00(42.5-82.0)$ & $0.720(0.553-0.852)$ & 0.0088 \\
MP-Hy & $>8.91$ & $78.57(49.2-95.3)$ & $72.00(50.6-87.9)$ & $0.800(0.641-0.911)$ & 0.0001 \\
P-MP & $\leq 36.75$ & $35.71(12.8-64.9)$ & $92.00(74.0-99.0)$ & $0.637(0.468-0.785)$ & 0.1718 \\
Ratio 1 & $>0.2108$ & $50.00(23.0-77.0)$ & $100.00(86.3-100.0)$ & $0.789(0.628-0.903)$ & 0.0003 \\
Ratio 2 & $\leq 0.7158$ & $64.29(35.1-87.2)$ & $92.00(74.0-99.0)$ & $0.840(0.687-0.937)$ & $<0.0001$ \\
Ratio 3 & $>0.2771$ & $57.14(28.9-82.3)$ & $96.00(79.6-99.9)$ & $0.806(0.648-0.915)$ & $<0.0001$ \\
\hline
\end{tabular}

Area under the ROC curve using to determine cut-off point of factor's in OSAS. Sen: Sensitivity, Spe: Specificity, AUC: area under the curve 
dicates that ratio parameters were more useful in predicting moderate-severe OSAS subjects than absolute values.

\section{DISCUSSION}

The gold standard for the diagnosis of OSAS in children remains attended overnight polysomnography (PSG). However, in the United States, only $10 \%$ of children are treated for sleep-disordered breathing based on a PSG result. ${ }^{9)}$ Many otolaryngologists decide on adenotonsillectomy based on adenotonsillar size or medical history provided by the caregiver. Even in adults, tonsil size may influence the severity of OSA. ${ }^{10)}$ However, it is quite difficult to predict the presence or severity of OSAS on the basis of physical examinations of tonsil and adenoid size. ${ }^{11)}$ In this study, physical parameters (tonsil size and adenoid ratio) did not show differ between the control group and the OSAS group, and even after additional grouping according to OSAS severity, there were no statistically significant differences in physical parameters (Table 5). This indicates that a simple physical examination may not be sufficient to predict the existence of OSAS. A questionnaire for OSAS has also been used, but the results in terms of diagnostic accuracy are inconsistent. ${ }^{12)}$

Cephalometric analysis is a simple, objective, and noninvasive method for facial skeletal, orthodontic assessment related to OSAS. Various parameters from cephalometric images have been measured and used to assess pediatric subjects suspected of having OSAS. Although these parameters are reportedly related to OSAS, they can be difficult to directly apply to each patient. Thus, in this study, we sought to find a simple and intuitive cephalometric parameter associated with OSAS.

As mentioned above, human laryngeal descent is a part of human evolution involving phonation and speech, resulting in a long, narrow, and collapsible upper airway. Elongation of the larynx might be associated with a higher risk of OSAS. ${ }^{13)}$ In previous adult studies, Yoshihiro et al. used CT scans in Japanese adult patients to demonstrate that airway length from the posterior end of the hard palate to the vocal cord that was significantly associated with AHI. ${ }^{5)}$ Similarly, Segal et al. used CT scans to show that the distance between the posterior part of the hard palate bone to the upper posterior part of the hyoid bone significantly correlated with AHI in adults $(\mathrm{r}=0.406, \mathrm{p}=0.049) .{ }^{14)}$ In cephalometric analyses of adults, similar results have been reported. ${ }^{15)}$ According to these reports, it is possible that upper airway length is associated with severity of OSAS.

However, in pediatric subjects, it is not easy to justify CT scans due to the relatively high radiation exposure. Thus, lateral cephalometry is commonly used to evaluate upper airway length. Because lateral cephalometry may not be reliable in identifying the precise location of the epiglottis or vocal cord, in this study, we used the hyoid bone and posterior nasal spine (PNS) as landmarks.

In our results, similar to those found in adults, the upper airway length (P-Hy) was somewhat longer with increasing OSAS severity. Although the OSAS group was younger than the control group, the absolute length of the upper airway was longer in the OSAS group than in the control group, supporting the fact that a longer airway might be associated with a higher risk of OSAS. However, there are also some reports contrary to our results. For example, the study in children with OSA using magnetic resonance imaging was reported by Arens et al. ${ }^{16)}$ They reported that the mean cross-sectional area of the total airway, minimal crosssectional area, and airway volume were significantly small-

Table 7. The univariable logistic regression analysis of each variable

\begin{tabular}{lccccc}
\hline & & $\mathrm{AHI} \leq 5(\mathrm{n}=25,64.1 \%)$ & $\mathrm{AHI}>5(\mathrm{n}=14,35.9 \%)$ & Odds ratio $(95 \% \mathrm{Cl})$ & $\mathrm{p} \mathrm{value}$ \\
\hline Gender & Male & $15(60.0)$ & $11(78.6)$ & $2.444(0.542-11.028)$ & 0.2449 \\
Age & $<6$ & $6(24.0)$ & $6(42.9)$ & $2.375(0.585-9.640)$ & 0.2263 \\
Tonsil & $>2$ & $19(76.0)$ & $13(92.9)$ & $4.104(0.441-38.221)$ & 0.2148 \\
AN-r & $>0.6$ & $8(32.0)$ & $11(78.6)$ & $7.791(1.690-35.918)$ & 0.0085 \\
P-HY & $>52.69$ & $9(36.0)$ & $11(78.6)$ & $6.518(1.432-29.666)$ & 0.0153 \\
Mp-HY & $>8.91$ & $7(28.0)$ & $11(78.6)$ & $9.427(2.008-44.259)$ & 0.0045 \\
P-MP & $\leq 36.75$ & $2(8.0)$ & $5(35.7)$ & $6.389(1.044-39.112)$ & 0.0448 \\
Ratio 1 & $>0.2108$ & $1(4.0)$ & $7(50.0)$ & $23.994(2.509-229.458)$ & 0.0058 \\
Ratio 2 & $\leq 0.7158$ & $2(8.0)$ & $9(64.3)$ & $20.700(3.381-126.720)$ & 0.0010 \\
Ratio 3 & $>0.2771$ & $1(4.0)$ & $8(57.14)$ & $32.000(3.329-307.633)$ & 0.0027 \\
\hline
\end{tabular}

Statistics were carried out using logistic regression 
er in the OSAS group, but that the upper airway length did not differ between the control and OSAS groups. These results might be because the authors did not conduct PSG in the control group, and subjects were only screened by medical history and questionnaire completion. In addition, subjects in the OSAS group were not divided according to severity, so the effects of OSA severity were not considered. In our study, the control group and the mild OSAS group did not show statistically significant differences among cephalometric parameters, and only the moderatesevere group showed differences in airway length compared to the control and mild groups.

Among the various cephalometric parameters measured, the lower segment of the upper airway (MP-Hy) showed a significant increase in the moderate-severe group, and there were no significant differences between the control and mild groups. The upper segment of the upper airway (PMP) did not show a significant change according to OSAS severity (Table 5). These results suggest that the distance of the lower segment of the upper airway (MP-Hy) might be an important parameter for discriminating the moderatesevere group from other groups, and hyoid bone position might be an important factor related to OSAS severity.

Similar results have been reported in other studies. For example, Vieira et al. reported that hyoid bone position was inferiorly positioned in pediatric subjects with OSAS, ${ }^{17)}$ and that the distances from the hyoid bone to the hard palate and mandibular plane were longer in OSAS patients compared to nasal breathing patients by analysis of lateral cephalometry. Ping-Ying Chiang et al. reported similar results in pediatric OSAS subjects in Taiwan. ${ }^{18)}$ The authors found that the retroglossal space and hyoid were the most important parameters in predicting OSAS, and most other cephalometric parameters did not differ among the control and OSAS groups.

Growth patterns in children vary by individual. That is, even among children who are the same age, skeletal growth differs from individual to individual. Compared to absolute values from cephalometric assessments, the ratios of the distances may be a more reliable and consistent index in children. As shown in our study, univariable logistic regression analysis demonstrated that the ORs of ratio parameters were higher than absolute length values in identifying moderate-severe OSAS patients (Table 7). In addition, ratios of cephalometric parameters showed low sensitivities, but high specificities, of $90 \%$ or more.
Our study had some limitation. First, the OSAS group was divided into two, rather than three subgroups according to AHI, because the number of patients in each OSAS subgroup was not even. Second, this study did not assess enough subjects to perform a multiple regression analysis. Third, in patients with obstructive sleep apnea during asleep, the movement of upper airway is different from that of the awoken state. $^{19)}$

\section{CONCLUSIONS}

Measurements of whole airway length and the lower part of the upper airway on cephalometric images may be valuable in identifying patients with moderate-severe OSA. Moreover, ratios of each cephalometric parameter may be more reliable and consistent indices than absolute length. The ratios measurement of cephalometric values may be a useful and simple method for identification of pediatric OSAS .

\section{Acknowledgments}

Statistical consultation was supported by Department of Biostatistics of the Catholic Research Coordinating Center.

\section{REFERENCES}

1) Marcus CL, Brooks LJ, Draper KA, Gozal D, Halbower AC, Jones $\mathrm{J}$, et al. Diagnosis and management of childhood obstructive sleep apnea syndrome. Pediatrics 2012;130:e714-55.

2) Lumeng JC, Chervin RD. Epidemiology of pediatric obstructive sleep apnea. Proceedings of the American Thoracic Society 2008; 5:242-52.

3) Tan HL, Gozal D, Kheirandish-Gozal L. Obstructive sleep apnea in children: a critical update. Nature and science of sleep 2013;5:10923.

4) De Luca Canto G, Singh V, Major MP, Witmans M, El-Hakim H, Major PW, et al. Diagnostic capability of questionnaires and clinical examinations to assess sleep-disordered breathing in children: a systematic review and meta-analysis. Journal of the American Dental Association (1939) 2014;145:165-78.

5) Yamashiro Y, Kryger M. Is laryngeal descent associated with increased risk for obstructive sleep apnea? Chest 2012;141:1407-13.

6) Fujioka M, Young LW, Girdany BR. Radiographic evaluation of adenoidal size in children: adenoidal-nasopharyngeal ratio. AJR Am J Roentgenol 1979;133:401-4.

7) Jeong JH, Guilleminault C, Park CS, Son HL, Lee HK, Hwang SH, et al. Changes in salivary cortisol levels in pediatric patients with obstructive sleep apnea syndrome after adenotonsillectomy. Sleep Medicine 2014;15:672-6.

8) Park CS, Guilleminault C, Park HJ, Cho JH, Lee HK, Son HL, et al. Correlation of salivary alpha amylase level and adenotonsillar hypertrophy with sleep disordered breathing in pediatric subjects. Journal of clinical sleep medicine: JCSM: official publication of the American Academy of Sleep Medicine 2014;10:559-66.

9) Mitchell RB, Pereira KD, Friedman NR. Sleep-disordered breath- 
ing in children: survey of current practice. The Laryngoscope 2006; 116:956-8.

10) Park JY, Choi SY, Lee JW, Ye MK, Shin SH. The Effect of Tonsil Size on the Obstructive Sleep Apnea in Adults. J Rhinol 2011;18(2): 102-6.

11) Nolan J, Brietzke SE. Systematic review of pediatric tonsil size and polysomnogram-measured obstructive sleep apnea severity. Otolaryngology--head and neck surgery: official journal of American Academy of Otolaryngology-Head and Neck Surgery 2011;144:844-50.

12) Abrishami A, Khajehdehi A, Chung F. A systematic review of screening questionnaires for obstructive sleep apnea. Canadian journal of anaesthesia=Journal Canadien D'anesthesie 2010;57:423-38.

13) Susarla SM, Thomas RJ, Abramson ZR, Kaban LB. Biomechanics of the upper airway: Changing concepts in the pathogenesis of obstructive sleep apnea. Int J Oral Maxillofac Surg 2010;39:1149-59.

14) Segal Y, Malhotra A, Pillar G. Upper airway length may be associated with the severity of obstructive sleep apnea syndrome. Sleep \& breathing=Schlaf \& Atmung 2008;12:311-6.

15) Susarla SM, Abramson ZR, Dodson TB, Kaban LB. Cephalometric measurement of upper airway length correlates with the presence and severity of obstructive sleep apnea. Journal of oral and maxil- lofacial surgery: official journal of the American Association of Oral and Maxillofacial Surgeons 2010;68:2846-55.

16) Arens R, McDonough JM, Corbin AM, Rubin NK, Carroll ME, Pack AI, et al. Upper airway size analysis by magnetic resonance imaging of children with obstructive sleep apnea syndrome. American Journal of Respiratory and Critical Care Medicine 2003;167:65-70.

17) Vieira BB, Itikawa CE, de Almeida LA, Sander HH, Aragon DC, Anselmo-Lima WT, et al. Facial features and hyoid bone position in preschool children with obstructive sleep apnea syndrome. European archives of oto-rhino-laryngology: official journal of the European Federation of Oto-Rhino-Laryngological Societies (EUFOS): affiliated with the German Society for Oto-Rhino-LaryngologyHead and Neck Surgery 2014;271:1305-9.

18) Ping-Ying Chiang R, Lin CM, Powell N, Chiang YC, Tsai YJ. Systematic analysis of cephalometry in obstructive sleep apnea in Asian children. Laryngoscope 2012;122:1867-72.

19) Lee KY, Lee CM, Choi YJ, Lee YJ, Jung SS, Kim JS. Comparison of Electron Beam Tomography and Multi Detector Computed Tomography for Dynamic Upper Airway Study in Snorers. J Rhinol 2004;11(1,2):57-61. 